\title{
Throughput Gains with Limited Feedback in Cooperative Relay Networks
}

\author{
Nasir Ahmed and Behnaam Aazhang \\ Department of Electrical and Computer Engineering \\ Rice University \\ email: \{nasir,aaz\}@ rice.edu
}

\begin{abstract}
In this work, we consider delay-constrained systems in cooperative relay networks, and derive methods to maximize the throughput when a limited feedback link to the transmitter nodes exist. We derive the optimal rate control policy, and show that with just a few bits of feedback, most of the gains of the perfect feedback rate control policy are achievable. We maximize the throughput for popular methods of transmission in a network such as relaying, routing, and direct transmission. It is observed that for a finite rate of feedback, the throughput maximizing outage probability can be relatively high. This suggests using higher rate codes, and allowing some outages in an effort to increase the overall throughput. Interestingly, for large average power constraints, power control is unnecessary to maximize the throughput. On the other hand, for small average power constraints, we show that power control is more important than rate control.
\end{abstract}

\section{INTRODUCTION}

In recent years, there has been a growing demand for providing new services in wireless networks with increasing throughput and reliability requirements. One method to enable such services is through cooperative transmission [1], which has shown the ability to improve the reliability of network transmissions, even with simple repetition based coding protocols [4]. As a result of the time varying nature of the fading process, which causes random fluctuations in the received power level, the attempted rate of transmission can exceed the instantaneous capacity of the channel, which results in an outage event. To combat the effect of outages, feedback information is sent to the transmitters to allow for adaptive communications. Feedback can be exploited to perform power or rate control, allowing for a dynamically changing power or coding rate, based on the instantaneous channel state. The concept of network power control was addressed in [2] for applications requiring constant transmission rates. In this work, we consider the problem of exploiting the feedback to increase the overall throughput.

Typically, the performance of systems in the block fading network environment can be characterized by the outage probability [8] and the delay-limited capacity [7]. However, due to the effect of outages, the effective data rate, or throughput, is less than the attempted transmission rate. Higher transmission rates leads to a higher frame error rate (FER), and low transmission rates translate to low FER's. The FER, which serves as a tight lower bound to the outage probability [9], needs to be balanced with the transmission rate to maximize the throughput. We consider the practical problem of throughput maximization, which more accurately reflects end-to-end performance than the rate of transmission.

In the network setting, we consider different possible methods of transmission: relay coding, routing, and direct transmission. Our main contributions are three-fold. First, we derive the optimal rate control procedure which maximizes the system throughput for the above mentioned transmission techniques. The algorithm is designed based on the rate of the feedback link and the target outage probability. Interestingly, it is observed that for a finite rate of feedback there is an optimal outage operating point that maximizes the throughput, which for a small rate of feedback, can be greater than $10 \%$. This suggests that minimizing the outage probability is unnecessary to maximize the throughput. In fact, for a finite rate of feedback, it is better to increase the coding rate and allow for some frame errors.

Second, we show that for a small rate of feedback, it is unnecessary to temporally vary both the rate and power. For small average power constraints, power control with rate selection suffices to maintain a high throughput. On the other hand, for large average power constraints, adapting the transmission rate while using a constant power is nearly as useful as variable rate/variable power transmission.

Finally, we show the power of relay coding over both routing and direct transmission in terms of achievable throughput. Decode and forward [5], one of several cooperative transmission techniques discussed in this work, is shown to have a tremendous increase in throughput over direct transmission. Furthermore, decode and forward provides large throughput gains over routing, suggesting the importance of collaboration between the transmitting nodes in a network. Our results on the throughput gains of decode and forward, along with the outage analysis of [3] suggest that decode and forward is an appropriate network code in the fading relay channel.

\section{COnClusions}

The details of this work will be presented at the conference. We will analyze the throughput of different network transmission protocols in the fading channel when a limited feedback link to the transmitters exist. We derive the optimal rate control algorithm that maximizes the throughput. We show that only a few bits of feedback are needed to realize tremendous increases in throughput. An interesting conclusion 
from this work is that the optimal operating point in terms of throughput is at a moderately high outage probability, which suggests using higher rate codes at the expense of frame errors. Furthermore, we show that power control is only necessary for small average powers, while rate control is sufficient when the average power constraint is large.

\section{REFERENCES}

[1] A. Sendonaris and E. Erkip and B. Aazhang, User Cooperation DiversityPart I: System Description, IEEE Transactions on Communications, vol. 51, no. 11, 1927-1938,Nov. 2003.

[2] N. Ahmed, M. Khojastepour, A. Sabharwal, and B. Aazhang Power Control with Finite Rate Feedback for Cooperative Relay Networks, Intern. Symposium on Inform. Theory and its Applications (ISITA), Oct. 10-13, Parma, Italy, 2004.

[3] N. Ahmed, M. Khojastepour, and B. Aazhang Outage Minimization and Optimal Power Control for the Fading Relay Channel, IEEE Information Theory Workshop (ITW), Oct. 24-29, San Antonio, TX, 2004.

[4] N. Laneman, D. Tse and G. Wornell "Coooperative Diversity in Wireless Networks: Efficient Protocols and Outage Behavior," IEEE Trans. on Info. Theory., vol. 50, no. 12, Dec. 2004.

[5] T.M. Cover and A. E. Gamal "Capacity theorems for the relay channel," IEEE Trans. on Info. Theory, vol. 25, no. 5, pp., 572-584, Sept 1979.

[6] S. Bhashyam, A. Sabharwal and B. Aazhang, "Feedback Gain in Multiple Antenna Systems," IEEE Trans. on Comm., vol. 50, no. 5, pp., 795-798, May 2002.

[7] S. Hanly, D. Tse Multi-Access Fading Channels: Part II: Delay-Limited Capacities, IEEE Transactions on Information Theory, v. 44, No. 7, Nov., 1998, pp. 2816-2831

[8] L. H. Ozarow, S. Shamai and A. D. Wyner, Information theoretic considerations for cellular mobile radio, IEEE Trans. on Vehicular Technology, vol. 43, num. 2, pp. 359-378, May 1994.

[9] R. Knopp and P. Humblet, On coding for the block fading channel, IEEE Transactions on Information Theory, v. 46, No. 7, Jan., 2000, pp. 189-205

[10] A. Goldsmith, The capacity of downlink fading channels with variable rate and power, IEEE Trans. on Vehicular Technology, v. 46, No. 3, Aug. 1997 , pp. $569-580$ 